\title{
CORRELAÇÃO DA PRIMIPARIDADE DE MÃES ADOLESCENTES COM SUAS FILHAS ADOLESCENTES GRÁVIDAS
}

\author{
CORRELATION OF MOTHERS WITH HER DAUGHTERS \\ PRIMIPARITY TEENS TEEN PREGNANCY
}

\author{
Glícia Campanharo Malheiros ${ }^{1}$, Hugo de Oliveira Freixo ${ }^{1}$, Annelise Maria de Oliveira Wilken de Abreu² \\ 1: Acadêmicos de Medicina da Faculdade de Medicina de Campos \\ 2: Doutora em Biociência e Biotecnologia na UENF, Docente da Faculdade de Medicina de Campos
}

\section{RESUMO}

Introdução: A gravidez na adolescência é um importante assunto de saúde pública devido à prevalência com que vem ocorrendo no Brasil e no mundo. Objetivos: Correlacionar fatores envolvidos na primiparidade de adolescentes com a primiparidade de suas respectivas mães, também adolescentes grávidas. Métodos: Estudo observacional transversal baseado em entrevista realizada com adolescentes grávidas - 10 a 19 anos - atendidas em maternidades de Campos dos Goytacazes-RJ e no CRTCA I, de fevereiro a agosto de 2014. Esta pesquisa atende a Resolução 196/96 do Conselho Nacional de Saúde relativo às pesquisas envolvendo Seres Humanos. Resultados: As adolescentes apresentaram um percentual bastante elevado de relação instável no período da primeira gravidez em contrapartida as mães apresentaram um percentual maior de relação estável. A escolaridade tanto das adolescentes quanto das mães estavam no nível de $1^{\mathrm{a}}$ a $4^{\mathrm{a}}$ série do ensino fundamental, prevalecendo o nível alfabetizada. $\mathrm{Na}$ ocupação predominava as atividades do lar. Com relação à menarca verificou-se que a tendência é ocorrer cada vez mais precocemente. Conclusões: Meninas que foram mães adolescentes mais tardiamente são filhas de mães que tiveram a primeira gestação após 19 anos. Em contrapartida, a maioria das meninas mães adolescentes mais precocemente são filhas de mães também adolescentes precoce. Isso reforça a validação do estudo, confirmando uma tendência ressaltada em trabalhos descritos anteriormente.

Palavras-chave: "adolescente", "gravidez na adolescência", "primiparidade".

\begin{abstract}
Introduction: Adolescent pregnancy is an important public health issue due to its prevalence in Brazil and worldwide. Objectives: To correlate factors involved in the primiparity of adolescents with the primiparity of their respective mothers, also pregnant adolescents. Methods: A cross-sectional observational study based on an interview with pregnant adolescents (10 to 19 years old) attended at maternities of Campos dos Goytacazes-RJ and at CRTCA I from February to August 2014. This research complies with Resolution 196/96 of the National Council related to research involving human beings. Results: Adolescents presented a fairly high percentage of unstable relationship in the period of the first pregnancy. On the other hand, mothers presented a higher percentage of stable relationship. The educational level of both adolescents and mothers were in the 1st to 4 th grade level of elementary education, with literacy level prevailing. In the occupation predominated the activities of the home. With regard to the menarche it was verified that the tendency is to occur more and more precociously. Conclusions: Girls who were teenage mothers later were daughters of mothers who had their first pregnancy after 19 years. In contrast, the majority of adolescent teenage mothers are precocious daughters of early teenage mothers. This reinforces the validation of the study, confirming a trend highlighted in previously described studies.

Keywords: "teenager", "teenage pregnancy", "primiparity".
\end{abstract}




\section{INTRODUÇÃO}

A gravidez na adolescência é tema contemporâneo, abordado por diferentes áreas do conhecimento. ${ }^{1}$ É também uma situação de risco psicossocial que pode ser reconhecida como problema para os adolescentes, que assim iniciam uma família não intencionada, sendo a adolescência um período com repercussões em vários setores do desenvolvimento humano, dentre eles a saúde sexual e reprodutiva. Período este também seguido de transformações corporais intensas e radicais, que caracterizam a puberdade., ${ }^{2,3}$

Segundo a Organização Mundial da Saúde (OMS), os limites da adolescência se estendem dos 10 aos 19 anos de idade $^{3}$, subdivididos em adolescentes menores (de 10 a 14 anos) e adolescentes maiores (de 15 a 19 anos). ${ }^{4} \mathrm{E}$, no Brasil, de acordo com a Lei ${ }^{\circ} 8.069$ de 13 de julho de 1990, disposta no Estatuto da Criança e do Adolescente, a adolescência compreende as idades entre 12 e 18 anos. $^{5}$

Considerando a adolescência como um período repleto de mudanças significativas, a maioria dos estudos aborda a gestação, parto e puerpério com enfoque nos riscos físicos, psicológicos e sociais para a adolescente, sem enfatizar os aspectos relacionados com a primiparidade e gravidez na adolescência de suas respectivas mães. ${ }^{2}$

A primeira experiência sexual vem acontecendo cada vez mais precocemente, ocorrendo entre 14 anos e 16 anos. ${ }^{3}$ De acordo com a Pesquisa Nacional de Demografia e Saúde realizada no Brasil em 1996, 33\% das mulheres entre 15 e 19 anos responderam que já tinham tido uma relação sexual, enquanto dados da Pesquisa Nacional de Demografia e Saúde da Criança e da Mulher de 2006 mostraram que esta proporção tinha aumentado para $55 \%$. Esse cenário ajuda a explicar o comportamento da fecundidade entre as adolescentes no Brasil, que aumentou nos anos 80 e $90 .^{6}$

A contribuição proporcional desse grupo para a taxa de fecundidade total vem apresentando um crescimento contínuo, aumentando de 17\% em 1996 para 23\% em 2006. Ou seja, em 2006, aproximadamente um em cada quatro nascimentos no Brasil ocorreu entre adolescentes de 15 e 19 anos, o que sugere um problema de saúde pública. Essa proporção é muito superior à mundial e ao que seria esperado para o Brasil, já que o nível educacional e o acesso aos métodos contraceptivos também têm crescido no país. ${ }^{6}$
O estudo desenvolvido por Vanda Terezinha Vasconcelos (no período de janeiro a abril de 1996), nas unidades de saúde do Centro de Saúde da Criança e Adolescente e no Centro Assistencial São José, de correlação entre primiparidade em mães e primiparidade de suas filhas adolescentes grávidas mostrou que a maioria das adolescentes grávidas são filhas de mães que também tiveram sua primeira gravidez na adolescência.

\section{OBJETIVOS}

O trabalho em questão teve como objetivo correlacionar fatores envolvidos na primiparidade de adolescentes com a primiparidade de suas respectivas mães, também adolescentes grávidas.

\section{MÉTODOS}

Trata-se de um estudo observacional transversal.

\section{ÁREA DE TRABALHO}

O município de Campos dos Goytacazes está localizado ao norte do Estado do Rio de Janeiro, a $289 \mathrm{Km}$ da capital possuindo 16 distritos com uma população estimada em 406.000 habitantes, assim distribuída: 363.210 na região urbana e 42.790 na região rural. Sua sede, a cidade de Campos dos Goytacazes é a terceira em importância econômica no estado, e se encontra às margens do Rio Paraíba do Sul.

\section{POPULAÇÃO / AMOSTRA CRITÉRIO DE INCLUSÃO}

A amostra estudada foi composta pelas adolescentes grávidas - de 10 a 19 anos - atendidas nas maternidades (Hospital dos Plantadores de Cana, Santa Casa de Misericórdia de Campos e Sociedade Portuguesa de Beneficência de Campos) e no Centro de Referência e Tratamento da Criança e do Adolescente (CRTCA I) do Município de Campos dos Goytacazes-RJ de fevereiro a agosto de 2014, que aceitarem participar da pesquisa. As adolescentes que não souberam responder as perguntas foram aceitas as respostas de suas respectivas mães.

Para cálculo do tamanho amostral verificouse que o número médio de atendimento mensal de adolescentes nas maternidades e no Serviço de Ginecologia do CRTCA I está em torno de 300 adolescentes. Assumiu-se para o cálculo da amostra um erro amostral de 5\% e um nível de confiança de 
$95 \%$ ficando a amostra composta por 169 adolescentes.

\section{CRITÉRIO DE EXCLUSÃO}

Foram excluídas do estudo as adolescentes grávidas que não residam no Município de Campos dos Goytacazes-RJ, aquelas que não souberam a respeito da primiparidade de suas mães ou as que se recusaram a participar da pesquisa.

\section{VARIÁ VEIS}

As variáveis coletadas referentes à população investigada foram: 1) variável de desfecho: ter mãe que também teve sua primiparidade na adolescência; 2) variáveis secundárias que representaram potenciais fatores de risco: idade cronológica, local de habitação, renda mensal familiar, relação conjugal, grau de escolaridade, ocupação, menarca, métodos contraceptivos e pré-natal.

\section{PROCEDIMENTOS / TÉCNICAS DE COLETA DE DADOS}

A coleta de dados foi realizada nas maternidades após o parto das adolescentes grávidas e no pré-natal do Serviço de Ginecologia para adolescentes do CRTCA I nos dias de atendimento ambulatorial de fevereiro a agosto de 2014.

A técnica para coleta de dados foi através de entrevista diretamente com a adolescente ou com sua mãe utilizando um protocolo estruturado que abordou questões como idade cronológica, local de habitação, renda mensal familiar, relação conjugal, grau de escolaridade, ocupação, menarca, métodos contraceptivos e pré-natal.

Nesta entrevista, atendendo a Resolução 196/ 96 do Conselho Nacional de Saúde (CNS) relativo às pesquisas envolvendo Seres Humanos, aquelas que aceitaram participar, a adolescente ou a sua mãe, foram amplamente esclarecidas sobre os objetivos da pesquisa assim como os benefícios e riscos envolvidos. A mãe ou a adolescente que concordaram em participar, assinaram o Termo de Consentimento Livre e Esclarecido (TCLE).

\section{RISCOS E BENEFÍCIOS}

Não houve riscos relacionados diretamente à pesquisa. Quanto aos benefícios, o conhecimento sobre a relação entre a gravidez na adolescência e a primiparidade das mães dessas adolescentes grávidas, que também estiveram grávidas na adolescência, permitiu conhecer a real situação sobre a sexualidade precoce e seus aspectos atuais.

\section{ANÁLISE DOS DADOS}

Os dados obtidos foram tabulados no Programa EPIDATA e apresentados em gráficos e tabelas elaboradas no Programa Excel. A análise dos dados foi estatística e apresentada em frequência de ocorrência de cada variável a ser expressa em números absolutos e percentual. Foi feito o cruzamento da variável de desfecho (ser adolescente grávida e ter mãe que engravidou na adolescência) com as variáveis secundárias (análise bivariada) e, em casos necessários, foi feita análise multivariada de regressão logística.

\section{RESULTADOS}

Dentre as conquistas femininas com o decorrer dos anos, a dissociação entre prática sexual, casamento e reprodução a partir da acessibilidade que as mulheres tiveram em relação aos métodos contraceptivos pode ser considerada um marco muito importante na construção de sua autonomia e no processo da liberdade reprodutiva. ${ }^{7}$

Entretanto, tais mudanças não foram acompanhadas por políticas públicas educacionais e de saúde direcionadas às necessidades vivenciadas pelo contexto sexual, o que colaborou para o aumento da gravidez na adolescência. ${ }^{8}$

Das 100 meninas entrevistadas, 51 (51\%) eram solteiras e 49 (49\%) eram casadas. Pouco mais da metade das adolescentes (51\%) não têm uma base familiar no momento do nascimento do filho, possivelmente restando para os familiares adjuntos (pais, avós, outros) o sustento da criança. Isso consome e desgasta as relações intrafamiliares. ${ }^{9}$

Da amostra desta pesquisa, 89 meninas ( $89 \%)$ afirmaram não ter nenhuma renda mensal. $\mathrm{O}$ fato de $89 \%$ delas não terem renda corrobora a teoria do desgaste familiar. Elas não terão auxílio do Instituto Nacional do Seguro Social (INSS) nos meses subsequentes e dependerão de alguém para suprir as necessidades imediatas da criança. ${ }^{10}$

Tinham o ensino fundamental incompleto no momento da pesquisa $65 \%$, e $19 \%$ tinham o segundo grau incompleto. Apenas 5\% haviam concluído os estudos. Devido $84 \%$ delas terem seus filhos no momento em que estão em sua fase de formação acadêmica básica, pode significar um aumento da taxa de evasão escolar. Isso perpetua o ciclo de 
pobreza em que estas meninas não farão o ensino técnico/superior e terão de vender a força de trabalho ou empreender em um ambiente hostil para o pequeno empreendedor como acontece no Brasil. ${ }^{11}$

Das mães das adolescentes grávidas, $49 \%$ declararam não ter uma fonte de renda. Outras $21 \%$ afirmaram receber menos que 1 (um) salário mínimo mensal. Para discussão dos dados acima: corrobora a explicação do desgaste familiar.

O trabalho em questão mostrou que $24 \%$ das mães das adolescentes têm cinco filhos ou mais e 57\% das mães tem o ensino médio incompleto.

Meninas que se tornaram mães aos 13 anos (04), são filhas de mães primíparas aos 16 (01) e 19 (02) anos. Uma (01) menina grávida nessa faixa etária ( $25 \%$ do total) é filha de mãe com 20 anos ou mais.

Meninas que se tornaram mães aos 14 anos (13), são filhas de mães primíparas aos $12(01)$, 13(02), 15 (01), 16 (04), 17 (02), 18 (01) e 19 (01) anos. Uma (01) menina grávida nesta faixa etária (aproximadamente $8 \%$ do total) é filha de mãe com 20 anos ou mais.

Meninas que se tornaram mães aos 15 anos (19), são filhas de mães primíparas aos 14 (02), 15 (02), 16 (03), 17 (02), 18 (02) e 19 (02) anos. Seis (06) meninas grávidas nesta faixa etária (aproximadamente $32 \%$ do total) são filhas de mães com 20 anos ou mais.

Meninas que se tornaram mães aos 16 anos (32), são filhas de mães primíparas aos 13 (01), 14 (01), 15 (04), 16 (03), 17 (11), 18 (04) e 19 (03) anos. Cinco (05) meninas grávidas nesta faixa etária (aproximadamente 16\% do total) são filhas de mães com 20 anos ou mais.

Meninas que se tornaram mães aos 17 anos (12), são filhas de mães primíparas aos 15 (03), 16 (04), 17 (02) e 19 (02) anos. Uma (01) menina grávida nesta faixa etária (aproximadamente $8 \%$ do total) é filha de mãe com 20 anos ou mais.

Meninas que se tornaram mães aos 18 anos (12), são filhas de mães primíparas aos 16 (03), 17 (01) e 18 (01). Sete meninas grávidas nesta faixa etária (aproximadamente 58\% do total) são filhas de mães com 20 anos ou mais.

Meninas que se tornaram mães aos 19 anos (08), são filhas de mães primíparas aos 12 (01), 16 (01), 17 (01) e 19 (02) anos. Três meninas grávidas nesta faixa etária (aproximadamente $38 \%$ do total) são filhas de mães com 20 anos ou mais (Figuras 1 e 2).
Além disso, no trabalho realizado por Vanda Terezinha (1996), no item relação conjugal as adolescentes apresentavam um percentual bastante elevado de relação instável no período da primeira gravidez em contrapartida as mães apresentavam um percentual maior de relação estável. A escolaridade tanto das adolescentes quanto das mães estavam no nível de $1^{\mathrm{a}}$ a $4^{\mathrm{a}}$ série do ensino fundamental, prevalecendo o nível alfabetizada. $\mathrm{Na}$ ocupação predominava as atividades do lar. Com relação à menarca verificou-se que a tendência é ocorrer cada vez mais precocemente.

Talvez o aspecto mais proeminente dos dados que relatamos seja a constatação de que as características de inserção socioeconômica aparecem associadas com diferenças marcantes nas características das repercussões da gravidez adolescente. $^{12}$

Tal constatação sugere que as repercussões de uma gravidez adolescente não devem ser pensadas, exclusivamente, a partir do conjunto de exigências e responsabilidades impostas a quem a vivencia, exigindo a compreensão de como características do contexto cultural e socioeconômico em que a família (ou similar) da grávida está imersa modulam tais repercussões.

Essa modulação apresentou-se como sendo de forte determinação em relação aos aspectos fundamentais para a inserção social: escolarização e trabalho. ${ }^{12}$

Dez ou mais anos após a gravidez adolescente, todas as entrevistadas de famílias de baixa renda, continuam vivendo em famílias (a mesma de origem ou a sua própria família, com cônjuge e filhos) que permanecem sendo de baixa renda, o que as torna, de forma agravada (pelo alto número de filhos, pelas dificuldades de trabalhar, pelos reflexos negativos que a ignorância e a defasagem na compreensão da realidade social têm sobre a formação dos filhos), protagonistas de um evidente processo de exclusão. ${ }^{13}$

\section{CONCLUSÕES}

Meninas que foram mães adolescentes mais tardiamente são filhas de mães que tiveram a primeira gestação após 19 anos. Em contrapartida, a maioria das meninas mães adolescentes mais precocemente são filhas de mães também adolescentes precoce. Isso reforça a validação do estudo, confirmando uma tendência ressaltada em trabalhos descritos anteriormente. 


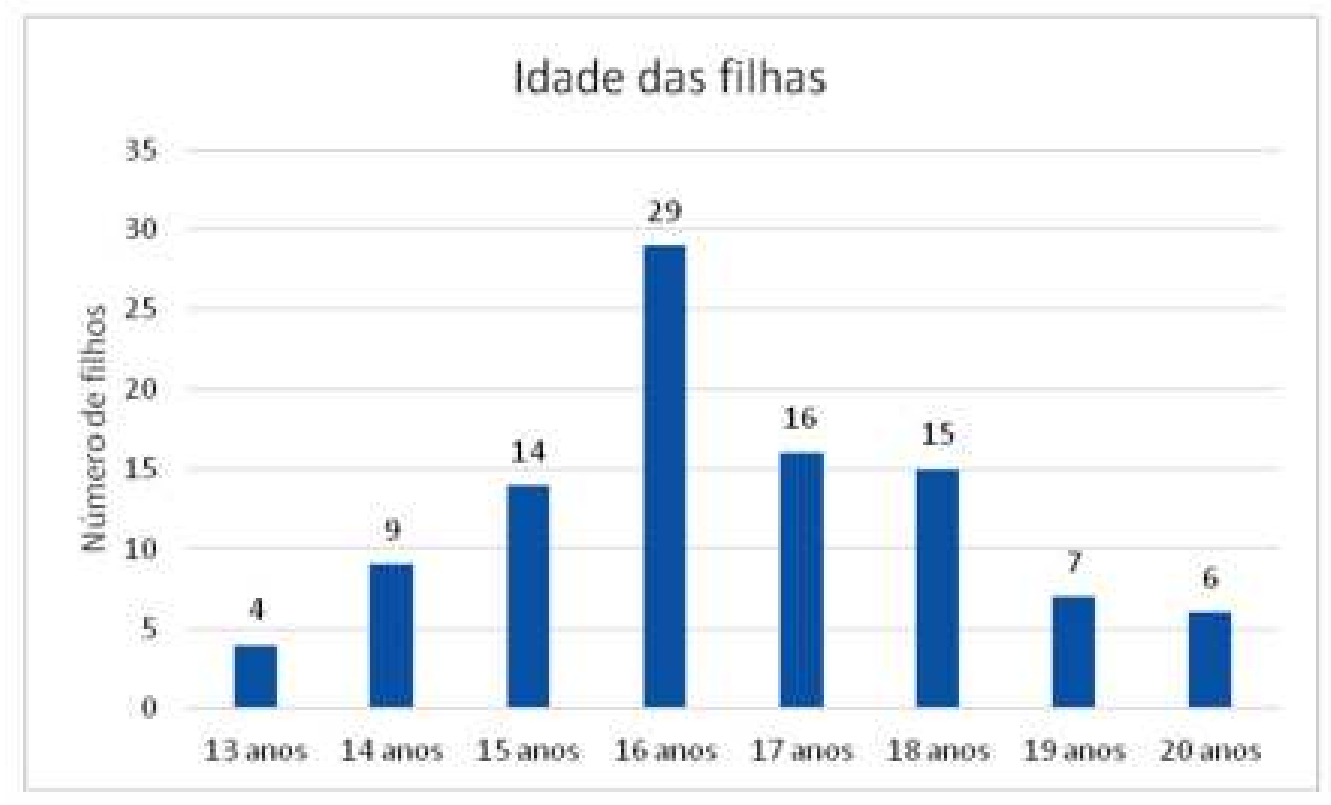

Figura 1: Número de filhos por idade.

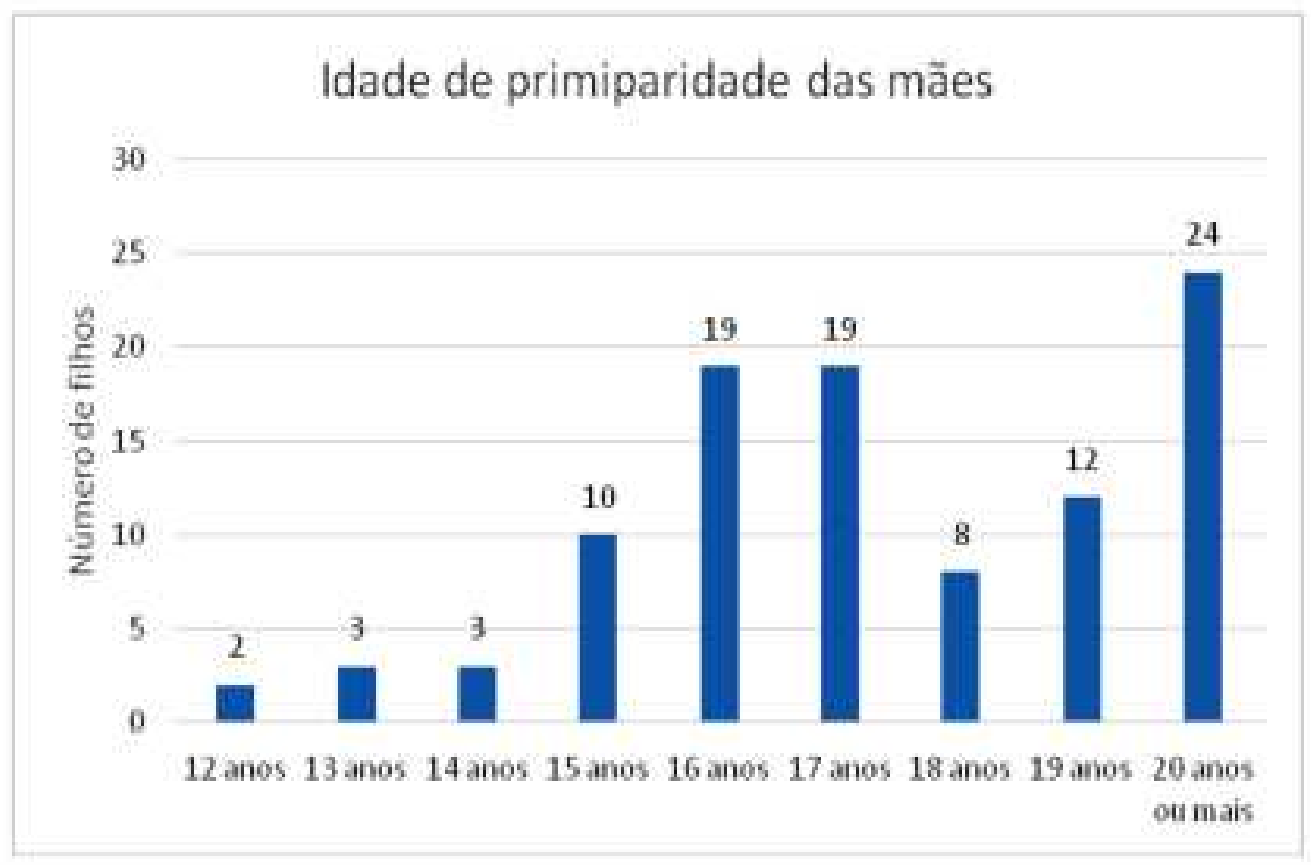

Figura 2: Nümero de filhos de acordo com a idade de primiparidade.

\section{REFERÊNCIAS BIBLIOGRÁFICAS}

1. FERREIRA, R. A.; FERRIANI, M. G. C.; MELLO, D. F.; CARVALHO, I. P.; CANO, M. A.; OLIVEIRA, L. A. Análise espacial da vulnerabilidade social da gravidez na adolescência. Cadernos de Saúde Pública, v. 28, n. 2, p. 313-23, 2012.

2. ENDERLE, C. F.; KERBER, N. P. C.; SUSIN, L. R. O.; SASSI, R. A. M. Avaliação da atenção ao parto por adolescentes em um hospital universitário. Revista Brasileira de Saúde Materno Infantil, v. 12, n. 4, p. 383-94, 2012.

3. VIEIRA, L. M.; GOLDBERG, T. B. L.; SAES, S. O.; DÓRIA, A. A. B. Abortamento na adolescência: da vida à experiência do colo vazio - um estudo qualitativo. Ciência \& Saúde Coletiva, v. 15, n. 2, p. 3149-56, 2010. 
4. BERETTA, M. I.; FREITAS, M. A.; DUPAS, G.; FABBRO, M. R. C.; RUGGIERO, E. M. S. A construção de um projeto na maternidade adolescente: relato de experiência. Revista da Escola de Enfermagem da USP, v. 45, n. 2, p. 533-6, 2011.

BRASIL. Estatuto da criança e do adolescente: Lei federal nº 8069, de 13 de julho de 1990. Rio de Janeiro: Imprensa Oficial, 2002.

5. VERONA, A. P. A.; JÚNIOR, C. S. D. Religião e fecundidade entre adolescentes no Brasil. Revista Panamericana de Salud Pública, v. 31, n. 1, p. 25-31, 2012.

6. NUNES, A. R. C.; OLIVEIRA, A. M.; ROZENO, F. S.; SILVIA, M. C.; GAZOLA, V. Gravidez na adolescência: fatores determinantes, ações preventivas. Centro Estadual de Educação Tecnológica Paula Souza - ETEC Prof. Mário Antônio Verza, Palmital, 2012.

7. PARIZ, J.; MENGARDA, C. F.; FRIZZO, G. B. A atenção e o cuidado à gravidez na adolescência nos âmbitos familiar, político e na sociedade: uma revisão da literatura. Saude soc, v. 21, n. 3, p. 623-36, 2012.

8. JAGER, M. E.; BOTTOLI, C. Paternidade: vivência do primeiro filho e mudanças familiares. Psicol. teor. Prat, v.12, n. 1, p. 141-53, 2011.

9. ROQUE, D. M.; FERREIRA, M. A. O que realmente importa em programas de transferência condicionada de renda? Abordagens em diferentes países. Saude soc, v. 24, n. 4, p. 1193-207, 2015.

10. SILVA, G. P. Análise de evasão no ensino superior: uma proposta de diagnóstico de seus determinantes. Avaliação: Revista da Avaliação da Educação Superior (Campinas), v. 18, n. 2, p. 311-33, 2013.

11. FISHER, M.; SHLOMO, I. B.; SOLT, I.; BURKE, Y. Z. Pregnancy Prevention and Termination of Pregnancy in Adolescence: Facts, Ethics, Law and Politics. Isr Med Assoc J, v. 17, n. 11, p. 665-8, 2015.

12. HARVILLE, E. W.; MADKOUR, A. S.; XIE, Y. Personality and adolescent pregnancy outcomes. J Adv Nurs, v. 71, n. 1, p. $148-59,2015$. 